\title{
Growth and Development Analysis of Clusterbean [Cyamopsis tetragonaloba (L). taub.] Genotypes (gum) as Influenced by Plant Density and Bio-Inoculants
}

\author{
Shilpa V. Chogatapur* and H.T. Chandranath \\ Department of Agronomy College of Agriculture, Dharwad University of Agricultural Sciences, \\ Dharwad-580 005, Karnataka, India \\ *Corresponding author
}

\begin{tabular}{|c|c|}
\hline & A B S T R A C T \\
\hline Keywords & The field experiment was conducted under rainfed condition during late Kharif \\
\hline $\begin{array}{l}\text { AM fungi, } \\
\text { Bradyrizhobium, } \\
\text { Gum, Specific leaf } \\
\text { area, PSB. }\end{array}$ & $\begin{array}{l}\text { Agricultural Sciences, Dharwad to study the Response of Clusterbean genotypes } \\
\text { (gum) to plant density and bio-inoculants. The experimental field was laid out in } \\
\text { split-split plot design with three replications. On the basis of results obtained from } \\
\text { present investigation Gaurishankar - 9, genotype recorded higher absolute growth }\end{array}$ \\
\hline Article Info & rate, leaf area ratio and specific leaf area. HG-365 recorded higher leaf weight \\
\hline $\begin{array}{l}\text { Accepted: } \\
17 \text { October } 2017 \\
\text { Available Online: } \\
10 \text { December } 2017\end{array}$ & $\begin{array}{l}\text { growth rate, leaf area ratio. Spacing level of } 30 \times 10 \mathrm{~cm} \text { recorded higher crop } \\
\text { growth rate and specific leaf weight. Application of Bradyrizhobium }+ \text { PSB }+ \text { AM } \\
\text { fungi recorded higher absolute growth rate, relative growth rate and crop growth } \\
\text { rate. }\end{array}$ \\
\hline
\end{tabular}

\section{Introduction}

Clusterbean [Cyamopsis tetragonoloba (L.) Taub] or Guar is a drought tolerant legume and grown mainly kharif season in arid and semi-arid region of tropical India. Multiple uses of the clusterbean make it as an important component of cropping systems of the region. Of late, it has acquired the status of industrial crop because of high glactomanan content in the endosperm of its seed, which has multiple industrial uses and thus a main foreign exchange earner for the area (Rathore et al., 2007). Clusterbean seed is used as a concentrate for animals and for extraction of gum. Seeds of clusterbean contain 22-33\% gum (Choudhary et al., 2014). The gum has its use in several industries, viz. textiles, paper, petroleum, pharmaceuticals, food processing, cosmetics, mining explosives and oil drilling. In India it is cultivated over an area of 51.51 lakh ha with the production of 24.60 lakh tonnes with an average yield of $478.0 \mathrm{~kg}$ per ha (Anon., 2013). India is the leading producer of guar and guar gum in the world and its share is around 80 per cent world production. Bio fertilizers play important role in maintaining the long term soil fertility and sustainability. It may increase yield of crops by $10-30$ 
percent (Khandelwal et al., 2012). Recent investigations, in the cultivation of gum guar have demonstrated commercial possibilities of growing guar under rainfed conditions. This has opened up possibilities of growing new cash crops in the region, although it is yet to find acceptance as a part of the cropping system of northern dry zone of Karnataka, but no systematic work has been conducted on this aspect.

The research information on cultivable aspects is lacking and also performance of different genotypes and spacing levels, hence, there is a need to study the response of clusterbean genotypes (gum purpose) to plant density and bio-inoculants.

\section{Materials and Methods}

The experiment was conducted at Agricultural Research Station, Annigeri, UAS Dharwad during the late kharif seasons of 2014. The soil was clay having initial soil $\mathrm{pH}$ of 7.9 and organic carbon $0.49 \%$ and available $\mathrm{N}, \mathrm{P}$ and $\mathrm{K}$ of $220,21.87$ and $462 \mathrm{~kg} \mathrm{ha}^{-1}$ respectively. The field was prepared by employing one deep ploughing. The average rainfall of area was $665.9 \mathrm{~mm}$ but during 2014 a rainfall of $771.0 \mathrm{~mm}$ was received. The experiment was laid out in split -split plot design 3 replications.

Two genotypes (HG- 365 and Gaurishankar 9), two spacings levels of (30 x $10 \mathrm{~cm}$ and 45 $\mathrm{x} 15 \mathrm{~cm}$ ) were allotted to main plot, sub plot and four treatments of Bio inoculants (Bradyrhizobium. PSB, AM fungi and Bradyrhizobium + PSB +AM fungi) were allotted to sub sub plot randomly. The crop was sown on 15 July and harvested on 17 November. Seeds of cluster bean were treated with biofertilizers, Bradyrhizobium + PSB @ $750 \mathrm{~g} \mathrm{ha}^{-1}$ and AM fungi applied at rate of 50 $\mathrm{kg} \mathrm{ha}{ }^{-1}$. Five random plants were selected from each plot, excluding the border row, for taking observations. Five plants were separated into leaf, stem and reproductive parts and dried in an oven at $70^{\circ} \mathrm{C}$ until a constant weight is obtained. Total dry matter was calculated by adding the dry weights of different plant parts and expressed as grams per plant.

\section{Absolute growth rate}

Absolute growth rate (AGR) was calculated by using the formula proposed by Radford (1967) and expressed in gram per day per plant $\left(\mathrm{g} \mathrm{day}^{-1}\right.$ plant $\left.^{-1}\right)$.

$$
\mathrm{AGR}=\frac{\mathrm{W} 2-\mathrm{W} 2}{\mathrm{t} 2-\mathrm{t} 1}
$$

Where,

$\mathrm{W} 2$ and $\mathrm{W} 1=$ Dry weight of whole plant $(\mathrm{g})$ at time $\mathrm{t} 2$ and $\mathrm{t} 1$ respectively.

$\mathrm{t} 2$ and $\mathrm{t} 1=$ Time intervals (days).

\section{Crop growth rate}

Crop growth rate (CGR) is defined as the rate of dry matter produced per unit land area per unit time. It is worked out by the formula proposed by Watson (1952) and expressed as $\mathrm{g}$ per $\mathrm{cm}^{2}$ per day.

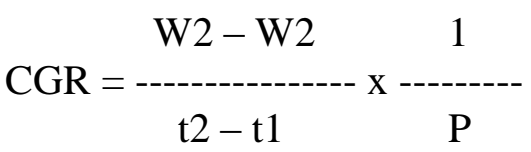

Where,

$\mathrm{W} 2$ and $\mathrm{W} 1$ = Dry weight of whole plant $(\mathrm{mg})$ at time $\mathrm{t} 2$ and $\mathrm{t} 1$ respectively.

$\mathrm{t} 2$ and $\mathrm{t} 1=$ Time intervals (days).

$\mathrm{P}=$ Land area $\left(\mathrm{cm}^{2}\right)$. 


\section{Relative growth rate}

Relative growth rate (RGR) is the rate of increase in dry weight per unit dry weight of the plant per unit time and expressed as g per $\mathrm{g}$ per day $\left(\mathrm{g} \mathrm{g}^{-1}\right.$ day $\left.^{-1}\right)$. It was calculated by the following formula proposed by Radford (1967).

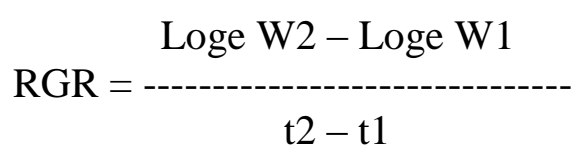

Where,

$\mathrm{W} 2$ and $\mathrm{W} 1$ = Dry weight of whole plant $(\mathrm{mg})$ at time $\mathrm{t} 2$ and $\mathrm{t} 1$ respectively.

$\mathrm{t} 2$ and $\mathrm{t} 1=$ Time intervals (days).

Loge $=$ Logarithm to base "e".

\section{Specific leaf area (SLA)}

Specific leaf area is the ratio of leaf area to leaf mass. It is a measure of relative spread of leaf. It is expressed in $\mathrm{cm}^{2} / \mathrm{g}$.

$$
\text { SLA }=\frac{\text { Leaf area }}{\text { Leaf dry weight }}
$$

\section{Specific leaf weight (SLW)}

The specific leaf weight is the ratio of leaf dry weight to leaf area. It indicates the leaf thickness and density and expressed as $\mathrm{g}$ $/ \mathrm{cm}^{2}$.

$$
\begin{gathered}
\text { Leaf dry weight } \\
\text { SLA }=\text {-af area }
\end{gathered}
$$

\section{Leaf area ratio (LAR)}

Leaf area ratio is the ratio of leaf area to total plant biomass. It is a measure of leafiness or photosynthetic surface relative to respiratory mass. It is expressed in $\left(\mathrm{cm}^{2} / \mathrm{g}\right)$

$$
\text { LAR }=\frac{\text { Leaf area of plant }}{\text { Total dry weight of plant }}
$$

\section{Leaf weight ratio (LWR)}

Leaf weight ratio is the ratio of mass of leaf to total dry mass of plant. It measure of allocation of leaf biomass

$$
\text { LWR }=\frac{\text { Mass of leaf }}{\text { Total mass of plant }}
$$

The data recorded on various parameters were subjected to Fisher's method of analysis of variance and interpretation of the data as given by Gomez and Gomez (1984).The level of significance used in ' $F$ ' and ' $\mathrm{t}$ ' test was $\mathrm{P}=$ 0.05 . Critical difference $(C D)$ values were calculated where the ' $F$ ' test was found significant. In case of non-significant effects, values of standard error of means alone were presented in Tables.

The mean value of sub sub plot and interaction were separately subjected to Ducan's multiple range test (DMRT) using the corresponding error mean sum of squares and degrees of freedom values.

\section{Results and Discussion}

Absolute growth rate (AGR) of clusterbean, genotypes was found significant at 30- 60 DAS (Table 1). Gaurishankar - 9 recorded significantly higher AGR compared to the genotype $\mathrm{HG}-365$. At 30-60 DA, a spacing of $45 \times 15 \mathrm{~cm}\left(0.57 \mathrm{~g} \mathrm{day}^{-1}\right.$ plant $\left.^{-1}\right)$ recorded significantly higher AGR when compared to at spacing of $30 \times 10 \mathrm{~cm}$. At 30 DAS application of Bradyrhizobium + PSB + AM fungi recorded significantly higher AGR $\left(0.59 \mathrm{~g} \mathrm{plant}^{-1}\right)$ when compared to other 
treatments. Relative growth rate (RGR) of clusterbean, genotypes was found significant at 30-60 DAS, a spacing level of $45 \times 15 \mathrm{~cm}$ $\left(0.026 \mathrm{~g} \mathrm{~g} \mathrm{~g}^{-1}\right.$ day $\left.^{-1}\right)$ recorded significantly higher RGR when compared to at spacing of $30 \times 10 \mathrm{~cm}\left(0.024 \mathrm{~g} \mathrm{~g}^{-1}\right.$ day $\left.^{-1}\right)$. Crop growth rate (CGR) of clusterbean at 30-60 DAS a spacing level of $30 \times 10 \mathrm{~cm}\left(0.0081 \mathrm{~g} \mathrm{~cm}^{-2}\right.$ day $^{-1}$ ) compared to at spacing of $45 \times 15 \mathrm{~cm}$ $\left(0.0039 \mathrm{~g} \mathrm{~cm}^{-2}\right.$ day $\left.^{-1}\right)$.
Leaf area ratio of clusterbean, genotypes was found significant at 90DAS, HG - 365 (18.88 $\mathrm{cm}^{2} \mathrm{~g}^{-1}$ ) recorded significantly higher leaf area ratio compared to the genotype Gaurishankar - $9\left(16.23 \mathrm{~cm}^{2} \mathrm{~g}^{-1}\right)$. Leaf weight ratio of clusterbean, genotypes was found significant at 90DAS, Gaurishankar - 9 (0.294 $\mathrm{cm}^{2} \mathrm{~g}^{-1}$ ) recorded significantly higher leaf weight ratio compared to the genotype $\mathrm{HG}-$ $365\left(0.260 \mathrm{~cm}^{2} \mathrm{~g}^{-1}\right)$.

Table.1 Absolute growth rate, relative growth rate and crop growth rate at different growth stages of cluster genotypes (gum) as influenced by plant density and bio-inoculants

\begin{tabular}{|c|c|c|c|c|c|c|c|c|c|}
\hline Treatments & $\begin{array}{l}\text { AGR 30- } \\
\text { 60DAS }\end{array}$ & $\begin{array}{l}\text { AGR 60- } \\
\text { 90DAS }\end{array}$ & $\begin{array}{l}\text { AGR } 90 \\
\text { DAS- } \\
\text { Harvest }\end{array}$ & $\begin{array}{l}\text { RGR 30- } \\
\text { 60DAS }\end{array}$ & $\begin{array}{l}\text { RGR 60- } \\
\text { 90DAS }\end{array}$ & $\begin{array}{l}\text { RGR } 90 \\
\text { DAS- } \\
\text { Harvest }\end{array}$ & $\begin{array}{l}\text { CGR 30- } \\
\text { 60DAS }\end{array}$ & $\begin{array}{l}\text { CGR 60- } \\
\text { 90DAS }\end{array}$ & $\begin{array}{l}\text { CGR 90- } \\
\text { Harvest }\end{array}$ \\
\hline \multicolumn{10}{|l|}{ Genotype } \\
\hline HG-365 & $0.44 \mathrm{~b}$ & $0.11 \mathrm{a}$ & $0.12 \mathrm{a}$ & $0.025 \mathrm{a}$ & $0.0028 \mathrm{a}$ & $0.0026 a$ & $0.0058 \mathrm{a}$ & $0.0006 \mathrm{a}$ & $0.0007 \mathrm{a}$ \\
\hline Gaurishankar-9 & $0.59 \mathrm{a}$ & $0.10 \mathrm{a}$ & $0.12 \mathrm{a}$ & $0.026 \mathrm{a}$ & $0.0020 \mathrm{a}$ & $0.0020 \mathrm{a}$ & $0.0061 \mathrm{a}$ & $0.0005 a$ & $0.0005 b$ \\
\hline S. Em \pm & 0.02 & 0.02 & 0.02 & 0.001 & 0.0004 & 0.0005 & 0.0003 & 0.0002 & 0.0001 \\
\hline \multicolumn{10}{|l|}{ Spacing } \\
\hline $30 \times 10 \mathrm{~cm}$ & $0.46 \mathrm{~b}$ & $0.11 \mathrm{a}$ & $0.12 \mathrm{a}$ & $0.024 \mathrm{~b}$ & $0.0026 \mathrm{a}$ & $0.0026 \mathrm{a}$ & $0.0081 \mathrm{a}$ & $0.0007 \mathrm{a}$ & $0.0009 a$ \\
\hline $45 \times 15 \mathrm{~cm}$ & $0.57 \mathrm{a}$ & $0.11 \mathrm{a}$ & $0.12 \mathrm{a}$ & $0.026 \mathrm{a}$ & $0.0023 \mathrm{a}$ & $0.0021 \mathrm{a}$ & $0.0039 \mathrm{~b}$ & $0.0003 \mathrm{~b}$ & $0.0003 b$ \\
\hline S. Em \pm & 0.02 & 0.01 & 0.02 & 0.0003 & 0.0002 & 0.0003 & 0.0001 & 0.0001 & 0.0001 \\
\hline \multicolumn{10}{|l|}{ Bio inoculants } \\
\hline Bradyrhizobium & $0.52 b$ & $0.10 \mathrm{a}$ & $21.87 \mathrm{a}$ & $0.025 \mathrm{a}$ & $0.0023 \mathrm{a}$ & $0.0023 a$ & $0.0059 \mathrm{a}$ & $0.0005 a$ & $0.0006 \mathrm{a}$ \\
\hline PSB & $0.48 \mathrm{~b}$ & $0.11 \mathrm{a}$ & $20.64 a$ & $0.025 \mathrm{a}$ & $0.0027 \mathrm{a}$ & $0.0028 \mathrm{a}$ & $0.0059 \mathrm{a}$ & $0.0006 \mathrm{a}$ & $0.0007 \mathrm{a}$ \\
\hline AM fungi & $0.46 \mathrm{~b}$ & $0.11 \mathrm{a}$ & $19.98 \mathrm{a}$ & $0.024 \mathrm{a}$ & $0.0027 \mathrm{a}$ & $0.0022 \mathrm{a}$ & $0.0057 \mathrm{a}$ & $0.0007 \mathrm{a}$ & $0.0006 \mathrm{a}$ \\
\hline $\begin{array}{l}\text { Bradyrhizobium + } \\
\text { PSB + AM fungi }\end{array}$ & $0.59 \mathrm{a}$ & $0.10 \mathrm{a}$ & $24.19 \mathrm{a}$ & $0.026 \mathrm{a}$ & $0.0020 \mathrm{a}$ & $0.0019 a$ & $0.0063 a$ & $0.0004 a$ & $0.0005 a$ \\
\hline S. Em \pm & 0.02 & 0.02 & 0.65 & 0.001 & 0.0005 & 0.0005 & 0.0002 & 0.0001 & 0.0001 \\
\hline
\end{tabular}

Table.2 Specific leaf area and specific leaf weight at different growth stages of cluster genotypes (gum) as influenced by plant density and bio-inoculants

\begin{tabular}{|c|c|c|c|c|c|c|c|c|}
\hline Treatments & $\begin{array}{l}\text { Specific leaf } \\
\text { area }\left(\mathrm{cm}^{2} / \mathrm{g}\right) \text { at } \\
30 \mathrm{DAS}\end{array}$ & $\begin{array}{l}\text { Specific leaf } \\
\text { area } \mathrm{cm}^{2} / \mathrm{g}^{\prime} \text { at } \\
60 \mathrm{DAS}\end{array}$ & $\begin{array}{l}\text { Specific leaf } \\
\text { area }\left(\mathrm{cm}^{2} / \mathrm{g}\right) \text { at } \\
90 \mathrm{DAS}\end{array}$ & $\begin{array}{l}\text { Specific leaf } \\
\text { area }\left(\mathrm{cm}^{2} / \mathrm{g}\right) \\
\text { at Harvest }\end{array}$ & $\begin{array}{c}\text { Specific leaf } \\
\text { weight }(\mathrm{g} \\
\left./ \mathrm{cm}^{2}\right) \text { at } 30 \\
\text { DAS }\end{array}$ & $\begin{array}{l}\text { Specific leaf } \\
\text { weight }(\mathrm{g} \\
\left./ \mathrm{cm}^{2}\right) \text { at } 60 \\
\text { DAS }\end{array}$ & $\begin{array}{l}\text { Specific leaf } \\
\text { weight }(\mathrm{g} \\
\left./ \mathrm{cm}^{2}\right) \text { at } 90 \\
\text { DAS }\end{array}$ & $\begin{array}{l}\text { Specific leaf } \\
\text { weight ( } \mathrm{g} \\
\left./ \mathrm{cm}^{2}\right) \text { at } \\
\text { harvest }\end{array}$ \\
\hline \multicolumn{9}{|l|}{ Genotype } \\
\hline HG-365 & $94.89 \mathrm{~b}$ & $85.07 \mathrm{a}$ & $66.74 a$ & $85.99 \mathrm{~b}$ & $0.0107 \mathrm{a}$ & $0.0120 \mathrm{a}$ & $0.0164 \mathrm{a}$ & $0.0119 \mathrm{a}$ \\
\hline Gaurishankar-9 & $102.59 \mathrm{a}$ & $87.02 \mathrm{a}$ & $65.35 \mathrm{~b}$ & $96.61 \mathrm{a}$ & $0.0100 \mathrm{~b}$ & $0.0117 \mathrm{a}$ & $0.0157 \mathrm{~b}$ & $0.0107 \mathrm{~b}$ \\
\hline S. Em \pm & 4.89 & 3.97 & 1.23 & 1.19 & 0.0005 & 0.0005 & 0.0001 & 0.0001 \\
\hline \multicolumn{9}{|l|}{ Spacing } \\
\hline $30 \times 10 \mathrm{~cm}$ & $95.82 \mathrm{~b}$ & $83.83 \mathrm{~b}$ & $65.83 \mathrm{a}$ & $87.85 \mathrm{~b}$ & $0.0107 \mathrm{a}$ & $0.0121 \mathrm{a}$ & $0.0163 \mathrm{a}$ & $0.0117 \mathrm{a}$ \\
\hline $45 \times 15 \mathrm{~cm}$ & $101.67 \mathrm{a}$ & $88.27 \mathrm{a}$ & $66.26 \mathrm{a}$ & $94.75 \mathrm{a}$ & $0.0100 \mathrm{~b}$ & $0.0116 \mathrm{~b}$ & $0.0159 \mathrm{~b}$ & $0.0109 \mathrm{~b}$ \\
\hline S. Em \pm & 3.04 & 1.37 & 1.43 & 2.48 & 0.0003 & 0.0002 & 0.0003 & 0.0003 \\
\hline \multicolumn{9}{|l|}{ Bio inoculants } \\
\hline Bradyrhizobium & $94.32 \mathrm{a}$ & $86.09 \mathrm{a}$ & $68.58 \mathrm{a}$ & $92.76 \mathrm{ab}$ & $0.0107 \mathrm{a}$ & $0.0118 \mathrm{a}$ & $0.0159 \mathrm{a}$ & $0.0110 \mathrm{ab}$ \\
\hline PSB & $97.66 \mathrm{a}$ & $84.00 \mathrm{a}$ & $62.24 \mathrm{a}$ & $83.63 b$ & $0.0104 \mathrm{ab}$ & $0.0124 \mathrm{a}$ & $0.0163 \mathrm{a}$ & $0.0123 \mathrm{a}$ \\
\hline AM fungi & $106.48 \mathrm{a}$ & $87.15 \mathrm{a}$ & $68.79 \mathrm{a}$ & $87.45 b$ & $0.0097 \mathrm{a}$ & $0.0116 \mathrm{a}$ & $0.0155 \mathrm{a}$ & $0.0116 \mathrm{ab}$ \\
\hline $\begin{array}{c}\text { Bradyrhizobium + } \\
\text { PSB + AM fungi }\end{array}$ & $96.50 \mathrm{a}$ & $86.95 \mathrm{a}$ & $64.58 \mathrm{a}$ & $101.36 \mathrm{a}$ & $0.0108 \mathrm{a}$ & $0.0117 \mathrm{a}$ & $0.0167 \mathrm{a}$ & $0.0103 \mathrm{~b}$ \\
\hline S. Em \pm & 4.99 & 3.44 & 4.11 & 4.13 & 0.0005 & 0.0005 & 0.0009 & 0.0006 \\
\hline
\end{tabular}


Table.3 Leaf area ratio and leaf weight ratio at different growth stages of cluster genotypes (gum) as influenced by plant density and bio-inoculants

\begin{tabular}{|c|c|c|c|c|c|c|c|c|}
\hline Treatments & $\begin{array}{l}\text { Leaf area } \\
\text { ratio }\left(\mathrm{cm}^{2} / \mathrm{g}\right) \\
\text { at } 30 \mathrm{DAS}\end{array}$ & $\begin{array}{l}\text { Leaf area } \\
\text { ratio }\left(\mathrm{cm}^{2} / \mathrm{g}\right) \\
\text { at } 60 \text { DAS }\end{array}$ & $\begin{array}{l}\text { Leaf area ratio } \\
\left(\mathrm{cm}^{2} / \mathrm{g}\right) \text { at } 90 \\
\text { DAS }\end{array}$ & $\begin{array}{l}\text { Leaf area } \\
\text { ratio }\left(\mathrm{cm}^{2} /\right. \\
\mathrm{g}) \text { at Harvest }\end{array}$ & $\begin{array}{l}\text { Leaf weight } \\
\text { ratio (g/palnt) } \\
\text { at } 30 \mathrm{DAS}\end{array}$ & $\begin{array}{l}\text { Leaf weight } \\
\text { ratio } \\
\text { (g/palnt) at } \\
60 \text { DAS }\end{array}$ & $\begin{array}{l}\text { Leaf weight } \\
\text { ratio (g/palnt) } \\
\text { at } 90 \mathrm{DAS}\end{array}$ & $\begin{array}{l}\text { Leaf weight } \\
\text { ratio (g/palnt) } \\
\text { at Harvest }\end{array}$ \\
\hline \multicolumn{9}{|l|}{ Genotype } \\
\hline HG-365 & $54.09 \mathrm{a}$ & $32.33 a$ & $16.23 b$ & $3.65 \mathrm{a}$ & $0.571 \mathrm{a}$ & $0.381 \mathrm{a}$ & $0.260 \mathrm{a}$ & $0.043 \mathrm{a}$ \\
\hline Gaurishankar-9 & $54.10 \mathrm{a}$ & $31.85 \mathrm{a}$ & $18.88 \mathrm{a}$ & $3.82 \mathrm{a}$ & $0.531 \mathrm{a}$ & $0.368 \mathrm{a}$ & $0.294 b$ & $0.040 \mathrm{a}$ \\
\hline S. Em \pm & 4.52 & 1.68 & 0.21 & 0.10 & 0.019 & 0.021 & 0.003 & 0.001 \\
\hline \multicolumn{9}{|l|}{ Spacing } \\
\hline $30 \times 10 \mathrm{~cm}$ & $52.31 b$ & $32.40 \mathrm{a}$ & $17.42 \mathrm{a}$ & $3.71 \mathrm{a}$ & $0.549 \mathrm{a}$ & $0.387 \mathrm{a}$ & $0.274 \mathrm{a}$ & $0.042 \mathrm{a}$ \\
\hline $45 \times 15 \mathrm{~cm}$ & $55.88 \mathrm{a}$ & $31.78 \mathrm{a}$ & $17.69 \mathrm{a}$ & $3.76 \mathrm{a}$ & $0.552 \mathrm{a}$ & $0.363 \mathrm{a}$ & $0.280 \mathrm{a}$ & $0.040 \mathrm{a}$ \\
\hline S. Em \pm & 1.58 & 1.77 & 0.48 & 0.10 & 0.004 & 0.015 & 0.011 & 0.001 \\
\hline \multicolumn{9}{|l|}{ Bio inoculants } \\
\hline Bradyrhizobium & $53.13 \mathrm{a}$ & $32.87 \mathrm{a}$ & $18.24 \mathrm{a}$ & $3.70 \mathrm{a}$ & $0.563 \mathrm{a}$ & $0.381 \mathrm{a}$ & $0.284 \mathrm{ab}$ & $0.040 \mathrm{~b}$ \\
\hline PSB & $53.70 \mathrm{a}$ & $30.70 \mathrm{a}$ & $16.76 \mathrm{a}$ & $3.78 \mathrm{a}$ & $0.550 \mathrm{a}$ & $0.371 \mathrm{a}$ & $0.269 \mathrm{ab}$ & $0.045 \mathrm{a}$ \\
\hline AM fungi & $56.11 \mathrm{a}$ & $31.55 \mathrm{a}$ & $16.95 a$ & $3.62 \mathrm{a}$ & $0.531 \mathrm{a}$ & $0.363 \mathrm{a}$ & $0.254 \mathrm{~b}$ & $0.042 \mathrm{ab}$ \\
\hline $\begin{array}{c}\text { Bradyrhizobium + } \\
\text { PSB + AM fungi }\end{array}$ & $53.43 \mathrm{a}$ & $33.24 \mathrm{a}$ & $18.27 \mathrm{a}$ & $3.84 \mathrm{a}$ & $0.558 \mathrm{a}$ & $0.384 \mathrm{a}$ & $0.301 \mathrm{a}$ & $0.039 \mathrm{~b}$ \\
\hline S. Em \pm & 2.27 & 1.22 & 0.93 & 0.17 & 0.013 & 0.011 & 0.012 & 0.001 \\
\hline
\end{tabular}

Specific leaf area of clusterbean, genotypes was found significant at 30DAS, Gaurishankar - $9\left(102.59 \mathrm{~cm}^{2} \mathrm{~g}^{-1}\right)$ recorded significantly higher specific leaf area compared to the genotype HG - 365 (94. 89 $\left.\mathrm{cm}^{2} \mathrm{~g}^{-1}\right)$, a spacing of $45 \times 15 \mathrm{~cm}\left(101.67 \mathrm{~cm}^{2}\right.$ $\left.\mathrm{g}^{-1}\right)$ recorded significantly higher specific leaf area when compared to at spacing of $30 \times 10$ $\mathrm{cm}\left(95.82 \mathrm{~cm}^{2} \mathrm{~g}^{-1}\right)$. At the time of harvest Gaurishankar - $9\left(96.61 \mathrm{~cm}^{2} \mathrm{~g}^{-1}\right)$ recorded significantly higher leaf specific area compared to the genotype $\mathrm{HG}-365$ (85.99 $\left.\mathrm{cm}^{2} \mathrm{~g}^{-1}\right)$, a spacing of $45 \times 15 \mathrm{~cm}\left(94.75 \mathrm{~cm}^{2}\right.$ $\left.\mathrm{g}^{-1}\right)$ recorded significantly higher leaf specific area when compared to at spacing of $30 \times 10$ $\mathrm{cm} \quad\left(87.85 \quad \mathrm{~cm}^{2} \quad \mathrm{~g}^{-1}\right)$. Application of Bradyrhizobium + PSB + AM fungi recorded significantly higher specific leaf area (101.36 $\mathrm{cm}^{2} \mathrm{~g}^{-1}$ ) when compared to other treatments at the time of harvest. Specific leaf weight of clusterbean, HG - $365\left(0.0107 \mathrm{~g} \mathrm{~cm}^{-2}\right)$ genotypes was found significant at 30DAS, recorded significantly higher specific leaf area compared to the genotype Gaurishankar - 9 $\left(0.0100 \mathrm{~g} \mathrm{~cm}^{-2}\right)$, a spacing of $30 \times 10 \mathrm{~cm}$ $\left(94.75 \mathrm{~g} \mathrm{~cm}^{-2}\right)$ recorded significantly higher specific leaf weight when compared to at spacing of $45 \times 15 \mathrm{~cm}\left(87.85 \mathrm{~g} \mathrm{~cm}^{-2}\right)$. At 90
DAS Specific leaf weight of clusterbean, HG - $365\left(0.0164 \mathrm{~g} \mathrm{~cm}^{-2}\right)$ genotypes was found significant recorded significantly higher specific leaf weight compared to the genotype Gaurishankar - $9\left(0.0157 \mathrm{~g} \mathrm{~cm}^{-2}\right)$, a spacing of $30 \times 10 \mathrm{~cm}\left(0.0163 \mathrm{~g} \mathrm{~cm}^{-2}\right)$ recorded significantly higher specific leaf weight when compared to spacing level of $45 \times 15 \mathrm{~cm}$ $\left(0.0159 \mathrm{~g} \mathrm{~cm}^{-2}\right)$. At the time of harvest, $\mathrm{HG}-$ $365\left(0.0107 \mathrm{~g} \mathrm{~cm}^{-2}\right)$ genotypes was found significant and recorded higher specific leaf weight compared to the genotype Gaurishankar - $9\left(0.0100 \mathrm{~g} \mathrm{~cm}^{-2}\right)$, a spacing of $30 \times 10 \mathrm{~cm}\left(94.75 \mathrm{~g} \mathrm{~cm}^{-2}\right)$ recorded significantly higher specific leaf weight when compared to at spacing of $45 \times 15 \mathrm{~cm}(87.85$ $\mathrm{g} \mathrm{cm}^{-2}$ ) (Tables 2 and 3).

Gaurishankar - 9 and spacing level of $45 \times 15$ $\mathrm{cm}$ recorded higher absolute growth rate. The spacing level of $30 \times 10 \mathrm{~cm}$ recorded crop growth rate at different growth stages. Gaurishankar - 9 genotype recorded higher leaf area ratio. Gaurishankar - 9 genotype recorded higher specific leaf area at different growth stages. The spacing level of $45 \times 15$ $\mathrm{cm}$ recorded significantly higher specific leaf area at different growth stages. HG- 
365genotype recorded higher specific leaf weight at different growth stages. The spacing level of $30 \times 10 \mathrm{~cm}$ recorded significantly higher specific leaf weight at different growth stages.

\section{References}

Anonymous, 2013, Ministry of Agriculture, Govt. of India. secy-agri@nic.in

Choudhary, R. S., Yadav, R. S. and Amin, A., 2014, Productivity and economics of clusterbean (Cyamopsis tetragonoloba) as influenced by phosphorus fertilization and biofertilizers in western Rajasthan. Ann. Agric. Res. New Series, 35(1): 62-64.
Gomez, K. A. and Gomez, A. A., 1984, Statistical Procedure for Agricultural Research. John Wiley and Sons, New Delhi, p. 680.

Radford, P.J., 1967, Growth analysis formulae, their use and abuse. Crop Sci., p. 171-175.

Rathore, V. S., Singh, J. P., Soni, M. L., Yadava, N. D. and Beniwal, R. K., 2007, Productivity, quality and resource utilization by clusterbean (Cyamopsis tetragonoloba) as influenced by nutrient management. Indian J. Agron., 52 (3): 243-246.

Watson, D. J., 1952. The physiological basis for variation in yield. Adv. Agron., 4: 101-145.

\section{How to cite this article:}

Shilpa V. Chogatapur and Chandranath, H.T. 2017. Growth and Development Analysis of Clusterbean (Cyamopsis tetragonaloba (L). taub.) Genotypes (gum) as Influenced by Plant Density and Bio-Inoculants. Int.J.Curr.Microbiol.App.Sci. 6(12): 2303-2308.

doi: https://doi.org/10.20546/ijcmas.2017.612.265 